\title{
Research Progress on Treatment of Abnormal Uterine Bleeding during Puberty with Traditional Chinese and Western Medicine
}

\author{
Dan Zhang1, Jing Cai ${ }^{2 *}$, Xiaoping Cui ${ }^{1}$, Xinchun Xiao ${ }^{1}$ \\ 1Shaanxi University of Chinese Medicine, Xianyang 712046, Shaanxi Province, China \\ 2The First Affiliated Hospital of Shaanxi University of Chinese Medicine, Xianyang 712000, Shaanxi Province, China \\ *Corresponding author: Jing Cai, 1308843516@qq.com
}

\begin{abstract}
In recent years, the frequency of clinical occurrence of abnormal uterine bleeding in adolescence has gradually increased. In order to fully understand the research progress in the treatment of abnormal uterine bleeding in adolescence, various studies and clinical experience have been reviewed by consulting related literature reports. The results found that currently Western medicine mainly uses hormone drugs in clinical treatment, but there are risks such as more clinical adverse reactions and higher recurrence rate; traditional Chinese medicine (TCM) treatment has achieved satisfactory results, but the advantages of sudden collapse and hemostasis are not obvious. Combined TCM and Western medicine treatment, it can learn from each other and has become a research hotspot in recent years, which is worthy of an in-depth discussion.
\end{abstract}

Keywords: Puberty; Abnormal uterine bleeding; Traditional Chinese and western medicine treatment

Publication date: July 2021; Online publication: July 31, 2021

\section{Introduction}

Abnormal uterine bleeding (AUB), is a common disease in clinical gynecology. It refers to bleeding from the uterine cavity that does not match any of the four elements of normal menstruation (cycle, menstruation, volume, regularity). Puberty is a special period of female development. The sign that a woman enters puberty is menstrual cramps. However, during this period, the hypothalamic-pituitary-ovarian gonadal axis has not yet matured and its regulatory function is not perfect, thus abnormal uterine bleeding is prone to occur. The majority of AUB in adolescence is Abnormal uterine bleeding due to ovulatory dysfunction (AUB-O). Domestic surveys show that $65 \%-90 \%$ of AUB in adolescence is due to the imperfect development of the hypothalamic-pituitary-ovarian axis, leading to dysfunctional and abnormal uterine bleeding ${ }^{[1]}$. In clinical research, patients with adolescent AUB should be diagnosed and treated efficiently, also managed appropriately for a long time. In recent years, the relevant research results of Chinese and Western medicine treatment of AUB in adolescence are reviewed to provide relevant reference for clinical diagnosis and treatment.

\section{TCM etiology and pathogenesis}

Abnormal uterine bleeding belongs to the category of "metrorrhagia" in TCM, which refers to menstruation that does not occur from time to time, or is dripping or an aggressive one. TCM believes that the main cause of AUB in adolescence is that adolescent girls have insufficient congenital endowment, childishness, and lack of charge. If you feel external pain at this time, it will easily cause damage to the charge and induce uterine bleeding. Deficiency can cause blood stasis, and blood stasis does not go away easily. This results 
in new blood being difficult to regenerate, blood does not follow the menstruation cycle, and it can also cause menstrual bleeding and uterine bleeding. In addition, adolescent girls have weak spleen and irregular diet, also since they are busy studying, overthinking, they tend to fails to make appointments, thus becoming ill. In addition, adolescent girls are more common in willful stubbornness, allowing for an easy move to stagnation of liver qi, and reduce of heat. Endogenous and scorching its impulse, blood heats rashly and causes uterine bleeding. In summary, the main pathogenic factors of AUB in adolescence are deficiency, heat, and blood stasis, also kidney deficiency is the root cause of the disease.

\section{TCM treatment}

"Danxi Xinfa Fuyu" puts forward three methods to cure bleeding, "Stop the flow, clear the source, and restore the old." The main therapies for adolescent AUB are tonifying the kidney, strengthening the Chong, and regulating the strength of the body. LiuXianming ${ }^{[2]}$ treated 63 cases of severe adolescence with AUBO. The bleeding phase prescription was supplemented with Juyuan decoction. After the bleeding was controlled, Bushen Guchong decoction was used to invigorate the spleen and kidney, and regulate the strength of the spleen and the body. The taste of the medicine was adjusted separately to focus on conditioning, and the effective rate was $93.7 \%$. Li Jin ${ }^{[3]}$ believes that AUB in adolescence is mostly a mixture of deficiency and excess, with more deficiency and less excess. The treatment is divided into two steps, where during bleeding, the method of invigorating the kidney, and using Gu Chong to stop the bleeding was used for medicinal purposes; in addition to Eclipta prostrata, Ejiao beads, big thistle, small thistle, Agrimony, Diyu charcoal, Panax notoginseng powder (Fenchong), Amomum villosum). After hemostasis, Zuogui pill was used to adjust and replenish Chong Ren, and the clinical effect was satisfactory.

\section{Western medicine etiology}

Adolescent AUB usually occurs 1-2 years after menarche, the main cause of the disease is that the hypothalamus and pituitary gland have not matured in adolescence, and the complete cycle regulation has not been established between the ovaries and the follicle stimulating hormone ${ }^{[4]}$ for follicle formation. The endometrium is long-term under the action of a single estrogen without progesterone antagonism, which causes estrogen withdrawal bleeding ${ }^{[5]}$.

\section{Western medicine treatment}

Western medical treatment of AUB can be divided into surgical treatment and drug treatment. Adolescent AUB patients are not suitable for surgical treatment, and clinical treatment is mainly hormone therapy. Pan Caixin ${ }^{[6]}$ treated with estrogen and progesterone sequential therapy after clinical diagnosis of AUB-O, initially given estradiol valerate and after 10 days, medroxyprogesterone acetate was, whereby the clinical effective rate was $95.1 \%$. Zhang Guilan ${ }^{[7]}$ clinically used desogestrel and ethinyl estradiol tablets to stop bleeding, and continued oral administration for 21 days. After the second half of the cycle, dydrogesterone tablets were added. The clinical bleeding time was significantly faster than the full cycle progesterone treatment. Sequential estrogen and progesterone therapy was administered according to the patient's ovarian endocrine status, which establishes a regular menstrual cycle, improves anovulatory uterine bleeding, and can also avoid excessive endometrial hyperplasia, that reduces bleeding recurrence after stopping the drug.

\section{Combined treatment of TCM and western medicine}

Traditional Chinese and Western medicine can be treated together and have complementary advantages, which is the trend of modern clinical research. Sun Dongli ${ }^{[8]}$ used drospirenone and ethinyl estradiol tablets (yousmin) to treat pubertal dysfunctional uterine bleeding by oral administration. Additionally, traditional 
Chinese medicine Zuogui pills, and its therapeutic effect was significantly better than that of the pure western medicine group. Yang Yanhong ${ }^{[9]}$ used Marvelon (desogestrel ethinyl estradiol) combined with Yunnan Hongyao capsules in the clinical treatment of anovulatory adolescent dysfunctional uterine bleeding, and the total clinical effective rate was $96.67 \%$.

\section{Discussion}

The pathogenesis of adolescent AUB is that the hypothalamus-pituitary-ovarian axis has not matured and is caused by insufficient hormone levels. Western medicine clinically treats adolescent AUB with hormone therapy. Estrogen can promote the growth of the endometrium, repair the wound of the endometrium and stop bleeding. Progesterone can change the endometrium from the proliferative phase to the secretory phase, shrink and fall off to adjust the menstrual cycle. However, in clinical trials, patients are prone to miss or mistake hormonal drugs, leading to menstrual cycle disorders. Traditional Chinese medicine believes that the main cause of AUB in adolescence is that the patient's congenital endowment is insufficient, the spleen and kidney are not in proper functioning state, and that it's not strong enough, causing the menstrual blood not to be restricted. The treatment is mainly to invigorate the kidney, adjust the menstrual cycle, and have fewer side effects, as well as low recurrence rate. Although TCM does not have obvious advantages in sudden collapse and hemostasis, the combination of Chinese and Western medicine can complement each other's advantages, quickly stop bleeding, improve the patient's anemia and hormone levels, as well as adjust the menstrual cycle. It is worthy of further exploration in clinical practice.

\section{Funding}

Scientific Research Project of Shaanxi Provincial Administration of Traditional Chinese Medicine (15LC007); Project title: Observation on the clinical efficacy of Shaanxi Province famous traditional Chinese medicine professor He Fengjie on the treatment of late menstruation with the method of nourishing the kidney and regulating the week.

Subject Innovation Team Project of Shaanxi University of Traditional Chinese Medicine(2019-YL08)

\section{Disclosure statement}

The author declares no conflict of interest.

\section{References}

[1] Jiang W, Xu J, Lin M, Ning L, 2018, Analysis of the Causes of Abnormal Uterine Bleeding in Women in Different Periods. Shaanxi Medical Journal, 47(07): 849-851.

[2] Liu X, 2005, Clinical Observation on 63 Cases of Severe Adolescent Dysfunctional Uterine Bleeding Treated by the Method of Invigorating the Spleen and Kidney. Clinical Journal of Traditional Chinese Medicine, (01): 34-35.

[3] Li J, 2007, Talking about the Treatment of Pubertal Metrorrhagia from the Kidney. Hebei Traditional Chinese Medicine, (05):438.

[4] Pan H, Wang X, Niu X, 2017, The Clinical Short-Term and Long-Term Curative Effect Study of 51 Cases of Spleen-Deficiency Adolescent Dysfunctional Uterine Bleeding Based on Syndrome Differentiation. World Traditional Chinese Medicine, 12(02): 343-347.

[5] Xie X, Kong B, Duan T, 2018, Obstetrics and Gynecology, 9 Edition. People's Medical Publishing House, Beijing, 334. 
[6] Pan C, 2019, The Value Analysis of Estrogen and Progesterone Sequential Therapy in the Treatment of Abnormal Uterine Bleeding in Adolescence. Electronic Journal of Practical Gynecology and Endocrinology, 6(09): 63-64.

[7] Zhang G, Hu X, Huang Z, 2021, Observation on the Effect of Desogestrel and Dydrogesterone in the Treatment of Abnormal Uterine Bleeding Caused by Ovulation Disorders in Adolescence. Chinese Journal of Maternal and Child Health Research, 32(01): 111- 114.

[8] Sun D, Lin B, Ma Y, et al., 2016, Analysis of Curative Effect of Integrated Traditional Chinese and Western Medicine on Adolescent Dysfunctional Uterine Bleeding. Journal of Liaoning University of Traditional Chinese Medicine, 18(01): 195-197.

[9] Yang Y, Guo M, Feng S, Yang F, 2020, Curative Effect Observation of Marvelon Combined with Yunnan Hongyao Capsules in the Treatment of Anovulatory Abnormal Uterine Bleeding in Adolescence. China Journal of Modern Medicine, 30(23): 51-54. 